\title{
Independent Prognostic Factors for Acute Organophosphorus Pesticide Poisoning
}

\author{
Weidong Tang, Feng Ruan MM, Qi Chen, Suping Chen, Xuebo Shao, Jianbo Gao MM, and \\ Mao Zhang MD
}

\begin{abstract}
BACKGROUND: Acute organophosphorus pesticide poisoning (AOPP) is becoming a significant problem and a potential cause of human mortality because of the abuse of organophosphate compounds. This study aims to determine the independent prognostic factors of AOPP by using multivariate logistic regression analysis. METHODS: The clinical data for 71 subjects with AOPP admitted to our hospital were retrospectively analyzed. This information included the Acute Physiology and Chronic Health Evaluation II (APACHE II) scores, 6-h post-admission blood lactate levels, post-admission 6-h lactate clearance rates, admission blood cholinesterase levels, 6-h postadmission blood cholinesterase levels, cholinesterase activity, blood $\mathrm{pH}$, and other factors. Univariate analysis and multivariate logistic regression analyses were conducted to identify all prognostic factors and independent prognostic factors, respectively. A receiver operating characteristic curve was plotted to analyze the testing power of independent prognostic factors. RESULTS: Twelve of 71 subjects died. Admission blood lactate levels, 6-h post-admission blood lactate levels, postadmission 6-h lactate clearance rates, blood $\mathrm{pH}$, and APACHE II scores were identified as prognostic factors for AOPP according to the univariate analysis, whereas only 6-h post-admission blood lactate levels, post-admission 6-h lactate clearance rates, and blood $\mathrm{pH}$ were independent prognostic factors identified by multivariate logistic regression analysis. The receiver operating characteristic analysis suggested that post-admission 6-h lactate clearance rates were of moderate diagnostic value. CONCLUSIONS: High 6-h post-admission blood lactate levels, low blood pH, and low post-admission 6-h lactate clearance rates were independent prognostic factors identified by multivariate logistic regression analysis. Key words: acute organophosphorus pesticide poisoning (AOPP); multivariate logistic regression analysis; independent prognostic factors; receiver operating characteristic curve. [Respir Care 2016;61(7):965-970. (C) 2016 Daedalus Enterprises]
\end{abstract}

\section{Introduction}

Since the 1980s, organophosphate compounds have constituted the pesticides most widely used for controlling

Mr Tang, Ms Q Chen, Ms S Chen, Mr Shao, and Mr Gao are affiliated with the Intensive Care Unit, First People's Hospital of Fuyang, Hangzhou, Zhejiang, China. Mr Ruan and Dr Zhang are affiliated with the Department of Emergency Medicine, the Second Affiliated Hospital of Medical College in Zhejiang University, Hangzhou, Zhejiang, China.

The authors have disclosed no conflicts of interest.

Correspondence: Weidong Tang, Intensive Care Unit, First People's Hospital of Fuyang, Hangzhou, Zhejiang 311400, China. E-mail: tangweidongmed@yeah.net.

DOI: $10.4187 /$ respcare. 04514 agricultural pests throughout the world. However, such compounds have a high potential for producing acute and sub-acute toxicities in humans. ${ }^{1}$ Due to their lack of regulation and easy availability in the developing world, especially Asian countries, acute organophosphorus pesticide poisoning (AOPP) continues to be a significant problem and a potential cause of human mortality. ${ }^{2}$ According to a report from the World Health Organization, AOPP has become the most common type of pesticide poisoning and is the cause of $\sim 3$ million human deaths worldwide each year. ${ }^{3}$ Presently, primary hospitals bear the brunt of this problem and treat hundreds of AOPP cases annually.

Prognostic factors are measurable characteristics associated with the risk of a subsequent outcome in people with a given disease or health condition, including simple measures, such as body mass index, and complex mea- 
sures, such as biomarkers. They can contribute to designing and analyzing trials ${ }^{4}$ and may even predict treatment response..$^{5}$ In AOPP, there are many factors that can be applied to detect poisoning severity and death.

Although numerous strategies have been utilized for detecting poisoning severity when exposed to organophosphate compounds, cholinesterase activity in plasma (or acetylcholinesterase activity in whole blood) is considered the preferred prognostic factor of acute organophosphorus poisoning, because it facilitates decision making in the duration of oxime therapy in cases of enzymes rapidly aging. ${ }^{6}$ However, in developing countries, this clinical decision is often delayed due to the lack of rapid laboratory diagnostic tools needed to confirm an AOPP diagnosis..$^{7-9}$ Additionally, scores of Acute Physiology and Chronic Health Evaluation II (APACHE II), a disease severity classification system, are positively correlated with disease severity and also are developing into a prognostic factor for adult patients with AOPP admitted to ICUs. ${ }^{10,11}$ Recently, the prognostic value of serum lactate levels has been reported as being independent of the underlying critical illness or the presence of shock or organ failure. ${ }^{12-15}$

However, the most effective method for diagnosing AOPP and the most important factor to consider when developing a prognosis for patients with AOPP remain unknown. We hypothesized that multivariable regression analysis would reveal clinical risk factors suitable for prognostication in organophosphate poisoning. Therefore, we conducted this study to determine the independent prognostic factors of AOPP by using multivariate logistic regression analysis.

\section{Methods}

\section{Ethics Statement}

The protocol for data collection of subjects with AOPP was approved by the Ethics Committee of the First People's Hospital of Fuyang, Zhejiang Province, China, and the project number was 09A19.

\section{Study Population}

This retrospective study analyzed 71 subjects admitted to the emergency department and subsequently the ICU of Fuyang hospital, Zhejiang Province, for AOPP between March 2009 and March 2013. Diagnoses of AOPP were made in accordance with national emergency schemes for the treatment of AOPP (GB7794-87) revised in 1987. The subjects were selected according to the following conditions. Patients with severe heart disease, heart failure, severe liver disease, kidney disease, diabetes with metformininduced lactic acidosis, or cancer were excluded from the study. Also excluded were patients who had received an intravenous injection of sodium acetate, sodium acetate

\section{QUICK LOOK}

\section{Current knowledge}

Due to the widespread use of organophosphate compounds, acute organophosphorus pesticide poisoning is becoming a significant problem and a potential cause of human mortality. To detect poisoning severity, numerous strategies have been devised. The recommended cholinesterase activity in plasma analysis has not been widely used because of the lack of rapid laboratory diagnostic tools. Recently, the prognostic value of serum lactate levels has been reported as being independent of the underlying critical illness.

\section{What this paper contributes to our knowledge}

With multivariate logistic regression analysis, high 6-h post-admission blood lactate levels, low blood $\mathrm{pH}$, and low post-admission 6-h lactate clearance rates were found to be independent prognosis factors for AOPP subjects. The post-admission 6-h blood lactate clearance rate with moderate diagnostic value was suitable for assessing the severity of AOPP and subject prognosis.

Ringer solution, or sodium lactate Ringer solution prior to or within $6 \mathrm{~h}$ after admission to the hospital, considering that the lactate in Ringer solution may interfere with the serum lactate levels and lactate clearance rate and worsen liver and kidney failure. We included subjects who either unintentionally or intentionally had consumed an organophosphorus pesticide or who had been previously exposed to an unknown dose of organophosphorus pesticide or provided incomplete data.

\section{Clinical Evaluation}

Each subject received a score based on their disease severity as determined by the APACHE II system. Analyses of blood lactate and cholinesterase levels, blood gases, blood $\mathrm{pH}$, base excess, and $\mathrm{HCO}_{3}^{-}$levels were performed in the clinical laboratory unit of our hospital. After admission, each subject's 6-h lactate clearance rate was calculated using the following equation: post-admission 6-h lactate clearance $=$ (admission blood lactate level $-6-\mathrm{h}$ post-admission blood lactate level)/admission blood lactate $\times 100 \%$. Cholinesterase activity was calculated as: cholinesterase activity $=$ admission blood cholinesterase level/4,500. Data including each subject's sex, age, time from poisoning to treatment, duration of hospitalization, duration of ICU stay, mechanical ventilation time, and survival status. Hospitalization expenses were also recorded. 
Table 1. Subject Clinical Characteristics and Survival Rates

\begin{tabular}{lcc}
\hline \hline & Survival $(n=59)$ & Death $(n=12)$ \\
\hline Male, $n(\%)$ & $38(40)$ & $6(50)$ \\
Age, mean \pm SD y & $50.2 \pm 19.1$ & $54.7 \pm 17.6$ \\
Time from poisoning to treatment, mean \pm SD (median) h & $4.48 \pm 3.65(3)$ & $3.62 \pm 3.01(2.5)$ \\
APACHE II score, mean \pm SD & $22.64 \pm 7.19$ & .45 \\
Admission lactate level, mean \pm SD mmol/L & $2.78 \pm 2.30$ & .45 \\
6-h lactate level, mean \pm SD mmol/L & $2.03 \pm 1.98$ & $5.43 \pm 2.41$ \\
6-h lactate clearance, mean \pm SD $\%$ & $2.78 \pm 2.30$ & $5.94 \pm 2.20$ \\
Admission cholinesterase level, mean \pm SD units/L & $1205.66 \pm 1581.37$ & $-18.07 \pm 31.37$ \\
6-h cholinesterase level, mean \pm SD units/L & $1127.58 \pm 1373.45$ & $556.75 \pm 539.42$ \\
Cholinesterase activity, mean \pm SD $\%$ & $26.79 \pm 35.14$ & $399.83 \pm 454.61$ \\
pH, mean \pm SD & $7.32 \pm 0.13$ & $12.37 \pm 11.99$ \\
Base excess, mean \pm SD mmol/L & $-6.50 \pm 8.21$ & $7.14 \pm 0.06$ \\
HCO ${ }_{3}^{-}$level, mean \pm SD mmol/L & $18.07 \pm 5.24$ & $-12.16 \pm 6.81$ \\
Duration of hospitalization, mean \pm SD h & $12.73 \pm 7.50$ & .003 \\
Duration of ICU, mean \pm SD h & $6.80 \pm 3.69$ & .18 \\
Mechanical ventilation time, mean \pm SD h & $4.89 \pm 3.22$ & .09 \\
Hospitalization expenses, mean \pm SD U.S. dollar & $4,344.60 \pm 3,294.16$ & .18 \\
& & .001 \\
\end{tabular}

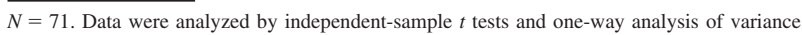

APACHE II = Acute Physiology and Chronic Health Evaluation II

\section{Statistical Analysis}

The enrolled subjects were assigned into 2 groups based on their clinical status. All study data were analyzed using SPSS 16.0 for Windows (SPSS, Chicago, Illinois). Values for continuous variables are expressed as the mean \pm SD. Values for categorical variables are expressed as percentages of the group from which they were derived. Independent variables included sex, age, time from poisoning to treatment, APACHE II scores, admission blood lactate levels, 6-h post-admission blood lactate levels, post-admission 6-h lactate clearance rates, admission blood cholinesterase levels, 6-h post-admission blood cholinesterase levels, cholinesterase activities, blood $\mathrm{pH}$, base excess levels, blood bicarbonate ion concentrations ( $\mathrm{HCO}_{3}^{-}$levels), duration of hospitalization, duration of ICU stay, mechanical ventilation times, and hospitalization expenses. The Kolmogorov-Smirnov method was used to test the normal distribution of each data set. Univariate analyses (the independent sample $t$ test and one-way analysis of variance) were used to assess relationships between the surveyed factors and AOPP. A stepwise multiple regression linear analysis was then used to identify significant prognostic factors for AOPP. Variables that were significantly associated with AOPP in the univariate analyses were selected as independent variables in the multivariate logistic regression analysis. Multivariate logistic regression analysis was used to provide a quantitative comparison of the relevance of putative prognostic factors, with the predictive ability of each factor indicated by an odds ratio (OR) and $95 \%$ CI. $P$ values $<.05$ were considered statistically significant. A receiver operating characteristic curve was plotted to analyze the independent prognostic factors and evaluate their usefulness.

\section{Results}

A total of 71 AOPP cases (subjects age 19-91 y, 44 males and 23 females) were retrospectively analyzed in this study. This total included 70 subjects who had intentionally consumed an organophosphorus pesticide and 1 subject with unintentional consumption. There were 31 cases of dichlorvos poisoning, 22 cases of methamidophos poisoning, 10 cases of rogor poisoning, 5 cases of triazophos poisoning, and 3 cases of unknown organophosphorus pesticide poisoning. The time periods from poisoning to treatment ranged from 0.5 to $16 \mathrm{~h}$. Although the majority of subjects either provided inaccurate information or failed to record information about the amount of pesticide consumed, all subjects underwent gastric lavage, catharsis, and diuretic treatment and were given atropine and cholinesterase agents. The comprehensive treatment also included monitoring and maintenance of life-sustaining organ functions, correction of acid/base imbalances, and provision of general clinical support. The overall subject mortality rate at the 21 st day after hospital admission was $16.9 \%$ (12 of 71). Subjects' clinical characteristics and survival rates are shown in Table 1. Our univariate analysis showed that prognostic factors for AOPP included subjects' admission blood lactate level, 6-h post-admission blood lactate level, post-admission 6-h lactate clearance rate, blood $\mathrm{pH}$, base excess, duration of hospitalization, and APACHE II score (all $P$ values $<.05$ ) (shown in Table 1 ). 
Table 2. Multivariate Logistic Regression Analysis of Acute Organophosphorus Pesticide Poisoning Prognostic Factors

\begin{tabular}{lrrrr}
\hline \hline & \multicolumn{1}{c}{$\beta$} & $P$ & $\begin{array}{l}\text { Odds } \\
\text { ratio }\end{array}$ & 95\% CI \\
\hline APACHE II score & 0.13 & .008 & 1.14 & $1.04-1.26$ \\
Admission lactate level, mmol/L & 0.37 & .005 & 1.45 & $1.12-1.87$ \\
6-h lactate level, mmol/L & 0.69 & $<.001$ & 1.99 & $1.36-2.91$ \\
6-h lactate clearance, \% & -2.74 & .003 & 0.065 & $0.01-0.40$ \\
pH & -10.70 & .001 & 0.00 & $0.00-0.01$
\end{tabular}

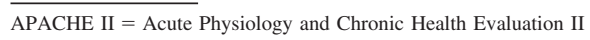

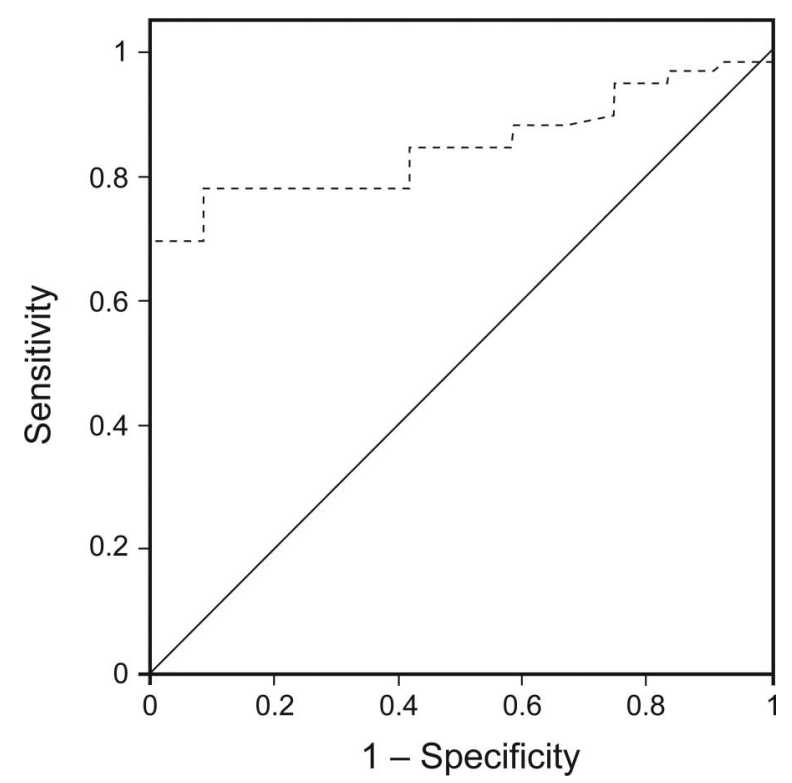

Fig. 1. Receiver operating characteristic analysis and curve for $6-\mathrm{h}$ lactate clearance after admission in predicting acute organophosphorus pesticide poisoning. Area under the curve $=0.85(95 \% \mathrm{Cl}$ 0.76-0.94), $P<.001)$.

Our multivariate logistic regression analysis showed that subjects' 6-h post-admission blood lactate level $(\mathrm{OR}=1.99$, $P<.001,95 \%$ CI 1.36-2.91), post-admission 6-h lactate clearance rate $(\mathrm{OR}=0.065, P=.003,95 \%$ CI $0.01-0.40)$, and blood $\mathrm{pH}$ level $(\mathrm{OR}=0.00, P=.001,95 \% \mathrm{CI}$ 0.00-0.01) were independent prognostic factors for AOPP (shown in Table 2). The receiver operating characteristic analysis showed a $5.99 \%$ cutoff point for post-admission 6-h lactate clearance rates in subjects with AOPP (area under the curve $=0.85,95 \%$ CI 0.76-0.94) $(P<.001)$, suggesting their moderate diagnostic value (Fig. 1).

\section{Discussion}

Acute organophosphorus pesticide poisoning is an important public health problem in developing countries. So an effective method for diagnosing AOPP and developing a prognosis for AOPP patients is a pressing need. Although a timely and accurate estimation of poisoning severity could reduce the number of deaths due to AOPP, no accurate criteria for determining this parameter have been identified. The aim of this study was to explore an effective method to detect poisoning severity. We introduced multivariate logistic regression to analyze the independent prognostic factors of AOPP.

In the study, diagnoses of AOPP were made in accordance with national emergency schemes for treatment of AOPP (GB7794-87) revised in 1987. Our multivariate logistic regression analysis identified 6-h post-admission blood lactate levels $(\mathrm{OR}=1.99, P<.001,95 \%$ CI $1.36-$ 2.91), post-admission 6 -h lactate clearance rates $(\mathrm{OR}=0.065, P=.003,95 \%$ CI 0.01-0.40), and blood $\mathrm{pH}$ $(\mathrm{OR}=0.00, P=.001,95 \% \mathrm{CI} 0.00-0.01)$ as independent prognostic factors for subjects with AOPP.

However, the butyrylcholinesterase activity in blood plasma (or acetylcholinesterase in whole blood) was not identified as an independent prognostic factor, Previously, it was recommended that an initial diagnosis of AOPP be confirmed by results of an assay that measures butyrylcholinesterase activity in blood plasma (or acetylcholinesterase in whole blood), $8,9,16$ since organophosphorus pesticides can inhibit cholinesterase activity and thus cause large amounts of acetylcholine to accumulate within the body. These accumulations result in cholinergic system dysfunction, hypoxia, inadequate tissue perfusion, microcirculation dysfunction, and disseminated intravascular coagulation, ultimately resulting in multiple organ dysfunction syndromes. ${ }^{8,9,17}$ However, the severity of early stage AOPP does not always correlate with changes in cholinesterase activity. ${ }^{18}$ A previous retrospective analysis of AOPP cases showed that changes in blood cholinesterase levels did not reflect the extent of clinical intoxication in 22 cases and should not be used as the sole reference when diagnosing and estimating AOPP severity. ${ }^{19}$ This finding was consistent with our current results, which showed no significant differences in admission cholinesterase levels, 6-h post-admission cholinesterase levels, and cholinesterase activity in the survival and non-survival groups $(P>.05)$.

Although recent advances in treating organophosphate compound-poisoned patients in the ICU have resulted in increased survival rates, such measures prolong a patient's ICU stay and increase hospital expenses. Providing patients with information regarding the severity of their illness upon ICU admission helps in determining the need for continuing expensive treatment and avoids unnecessary procedures. The prognostic APACHE II scoring system was developed to quantify a patient's severity of illness and likelihood of survival in an ICU population. This scoring system used a point score based on the initial values of 12 routine physiological measurements, includ- 
ing a patient's age and medical history, to provide a general measure of disease severity quantified within a range of $0-71 .{ }^{11}$ Furthermore, due to the strong and consistent relationship between acute physiological dysfunction and the risk of death caused by acute illness, ${ }^{11,20-22}$ this system can stratify a wide variety of patients according to their prognosis, and it was verified to be a useful prognostic indicator according to Kang et al. ${ }^{23}$ Although the difference in APACHE II scores in the survival group and nonsurvival group was significant in our study $(P<.05)$, the multivariate logistic regression analysis did not identify APACHE II score as an independent prognostic factor. This result suggested that it might be better to use a more useful method to assess the severity of organophosphate poisoning in ICU subjects. According to Hwang and coworkers, ${ }^{10}$ the Sequential Organ Failure Assessment was more useful, easier, and simpler than the APACHE II, so we suggest using the Sequential Organ Failure Assessment for related study in the future.

Global tissue hypoxia, accompanied by a myriad of pathogenic mechanisms that contribute to multi-system organ dysfunction syndrome, increases mortality, which results from the elevated blood lactate levels directly. ${ }^{24,25}$ Recently, blood lactate levels and lactate clearance rates have been recommended for use as biomarkers for mortality risk in subjects in the ICU.13-15,26-28 Interestingly, the prognostic value of lactate levels appears to be independent of the underlying critical illness and the presence of shock or organ failure. ${ }^{12-15}$ Although a high admission lactate level was previously identified as a significant independent predictor of mortality in adult subjects in the ICU, ${ }^{12,14}$ this finding was not replicated in other studies $^{13,15}$ and still requires more research to examine this factor. Lactate clearance refers to the rate of decline in blood lactate levels after the initiation of intensive medical care and has shown promise as a predictive indicator of patient death. 13,15,27 Two studies of adult subjects with shock showed that a lactate clearance rate of $<10 \%$ was related to an increased risk of mortality. ${ }^{13,28}$ Additionally, post admission 6-h lactate clearance was found to positively correlate with mortality in the pediatric ICU of our hospital, with a sensitivity of $75 \%$ and specificity of $97 \% .{ }^{27}$ Although the difference in admission lactate levels between the survival group and death group in our study was statistically significant $(P<.05)$, our multivariate logistic regression analysis showed that admission lactate levels were not an independent prognostic factor for survival. The receiver operating characteristic analysis showed a $5.99 \%$ cutoff point for post-admission 6-h lactate clearance rates (area under the curve $=0.85,95 \%$ CI $0.76-$ 0.94) $(P<.001)$, suggesting their moderate diagnostic value (see Fig. 1). The cutoff value for the receiver operating characteristic curve of post-admission 6-h lactate clearance rates seemed to be small, but the average of 6-h lactate clearance rates was negative; thus, a 6-h lactate clearance rate of $>5.99 \%$ might be a favorable factor for survival.

Still, there are many limitations in this study. The sample size is small, so the results cannot be generalized to all populations. Residual confounding is found in the statistical data, which may result in a misunderstanding of the data. Also, we are unable to control for the dose of organophosphate compound, which was different among subjects. Also, duration for treatment of organophosphate compound poisoning, which may be a key factor for death, was variable, with large SD values in both the survival and death groups, but the mean time and median time in the death group were less than that in the survival group; therefore, perhaps time alone is of little use in predicting survival. In this view, it is better to combine time and dose of organophosphate compound, although it would be better to control for time in further studies.

\section{Conclusions}

Subjects with AOPP with a high 6-h blood post-admission lactate level, low blood $\mathrm{pH}$ value, and low post-admission 6-h lactate clearance rate were found to be at an increased risk of death, and these were independent prognostic factors identified by multivariate logistic regression analysis. Furthermore, a patient's post-admission 6-h blood lactate clearance rate may be of great importance for assessing the severity of AOPP as well as the prognosis and possibly can be used as a screening tool to predict adverse outcomes.

\section{ACKNOWLEDGMENTS}

This study is based on data from the First People's Hospital of Fuyang and Second Affiliated Hospital. We are grateful for the assistance of the nursing, technical, administrative, and support staff in the ICUs and the Department of Emergency Medicine.

\section{REFERENCES}

1. Stephens R, Spurgeon A, Calvert IA, Beach J, Levy LS, Berry H, Harrington JM. Neuropsychological effects of long-term exposure to organophosphates in sheep dip. Lancet 1995;345(8958):1135-1139.

2. Eddleston M, Karalliedde L, Buckley N, Fernando R, Hutchinson G, Isbister G, et al. Pesticide poisoning in the developing world: a minimum pesticides list. Lancet 2002;360(9340):1163-1167.

3. Eddleston M, Phillips MR. Self poisoning with pesticides. BMJ 2004; 328(7430):42-44.

4. Maas AI, Marmarou A, Murray GD, Teasdale SG, Steyerberg EW. Prognosis and clinical trial design in traumatic brain injury: the IMPACT study. J Neurotrauma 2007;24(2):232-238.

5. Cianfrocca M, Goldstein LJ. Prognostic and predictive factors in early-stage breast cancer. Oncologist 2004;9(6):606-616.

6. Chowdhary S, Bhattacharyya R, Banerjee D. Acute organophosphorus poisoning. Clin Chim Acta 2014;431:66-76.

7. Eddleston M, Buckley NA, Eyer P, Dawson AH. Management of acute organophosphorus pesticide poisoning. Lancet 2008;371(9612): 597-607. 


\section{Acute Organophosphorus Pesticide Poisoning}

8. Hmouda H, Ben Salem C, Bouraoui K. Management of acute organophosphorus pesticide poisoning. Lancet 2008;371(9631):21692170; author reply 2170-2161.

9. Roberts DM, Aaron CK. Management of acute organophosphorus pesticide poisoning. BMJ 2007;334(7594):629-634.

10. Kim YH, Yeo JH, Kang MJ, Lee JH, Cho KW, Hwang S, et al. Performance assessment of the SOFA, APACHE II scoring system, and SAPS II in intensive care unit organophosphate poisoned patients. J Korean Med Sci 2013;28(12):1822-1826.

11. Knaus WA, Draper EA, Wagner DP, Zimmerman JE. APACHE II: a severity of disease classification system. Crit Care Med 1985; 13(10):818-829.

12. Smith I, Kumar P, Molloy S, Rhodes A, Newman PJ, Grounds RM, Bennet ED. Base excess and lactate as prognostic indicators for patients admitted to intensive care. Intensive Care Med 2001;27(1):74-83.

13. Nguyen HB, Rivers EP, Knoblich BP, Jacobsen G, Muzzin A, Ressler JA, Tomlanovich MC. Early lactate clearance is associated with improved outcome in severe sepsis and septic shock. Crit Care Med 2004;32(8): 1637-1642.

14. Jansen TC, van Bommel J, Schoonderbeek FJ, Sleeswijk Visser SJ, van der Klooster JM, Lima AP, et al. Early lactate-guided therapy in intensive care unit patients: a multicenter, open-label, randomized controlled trial. Am J Respir Crit Care Med 2010;182(6):752-761.

15. del Portal DA, Shofer F, Mikkelsen ME, Dorsey PJ Jr., Gaieski DF, Goyal M, et al. Emergency department lactate is associated with mortality in older adults admitted with and without infections. Acad Emerg Med 2010;17(3):260-268.

16. Thiermann H, Szinicz L, Eyer P, Zilker T, Worek F. Correlation between red blood cell acetylcholinesterase activity and neuromuscular transmission in organophosphate poisoning. Chem Biol Interact 2005;157:345-347.

17. Mizuno Y, Ohama E, Hirato J, Nakazato Y, Takahashi H, Takatama $\mathrm{M}$, et al. Nestin immunoreactivity of Purkinje cells in CreutzfeldtJakob disease. J Neurol Sci 2006;246(1):131-137.

18. Zhao D. [Characters of cholinesterase are correlated with acute organophosphorus pesticide poisoning]. Zhonghua Nei Ke Za Zhi 2000; 39(10):653-654
19. Qian XX. Investigation into the relationship between the extent of clinical intoxication and the change in blood cholinesterase at the early stage of acute organic phosphorus pesticide poisoning: clinical analysis of 96 cases. Chinese Crit Care Med 2001;39(10): 237-239.

20. Knaus WA, Wagner DP, Draper EA. Relationship between acute physiologic derangement and risk of death. J Chronic Dis 1985; 38(4):295-300.

21. Serpa Neto A, Assunção MS, Pardini A, Silva E. Feasibility of transitioning from APACHE II to SAPS III as prognostic model in a Brazilian general intensive care unit: a retrospective study. Sao Paulo Med J 2015;133(3):199-205.

22. Shoemaker WC, Chang P, Czer L, Bland R, Shabot MM, State D. Cardiorespiratory monitoring in postoperative patients: I. Prediction of outcome and severity of illness. Crit Care Med 1979;7(5):237242

23. Kang EJ, Seok SJ, Lee KH, Gil HW, Yang JO, Lee EY, Hong SY. Factors for determining survival in acute organophosphate poisoning. Korean J Intern Med 2009;24(4):362-367.

24. Karimova A, Pinsky DJ. The endothelial response to oxygen deprivation: biology and clinical implications. Intensive Care Med 2001; 27(1):19-31.

25. Rivers EP, Kruse JA, Jacobsen G, Shah K, Loomba M, Otero R, Childs EW. The influence of early hemodynamic optimization on biomarker patterns of severe sepsis and septic shock. Crit Care Med 2007;35(9):2016-2024.

26. Nguyen HB, Loomba M, Yang JJ, Jacobsen G, Shah K, Otero RM, et al. Early lactate clearance is associated with biomarkers of inflammation, coagulation, apoptosis, organ dysfunction and mortality in severe sepsis and septic shock. J Inflamm 2010;7:6.

27. Munde A, Kumar N, Beri RS, Puliyel JM. Lactate clearance as a marker of mortality in pediatric intensive care unit. Indian Pediatr 2014;51(7):565-567.

28. Arnold RC, Shapiro NI, Jones AE, Schorr C, Pope J, Casner E, et al. Multicenter study of early lactate clearance as a determinant of survival in patients with presumed sepsis. Shock 2009;32(1):35-39. 